OPEN ACCESS

Edited by:

Quanzi Li,

Chinese Academy of Forestry, China

Reviewed by:

Yuxiang Cheng,

Northeast Forestry University, China Keming Luo,

Southwest University, China

${ }^{*}$ Correspondence:

Juan Du

djuan@zju.edu.cn

tThese authors have contributed equally to this work

Specialty section:

This article was submitted to

Plant Biotechnology,

a section of the journal

Frontiers in Plant Science

Received: 30 April 2021

Accepted: 16 June 2021

Published: 12 July 2021

Citation:

An Y, Geng Y, Yao J, Wang $C$ and Du J (2021) An Improved

CRISPR/Cas9 System for Genome

Editing in Populus by Using Mannopine Synthase (MAS) Promoter.

Front. Plant Sci. 12:703546.

doi: $10.3389 / \mathrm{fp} / \mathrm{s} .2021 .703546$

\section{An Improved CRISPR/Cas9 System for Genome Editing in Populus by Using Mannopine Synthase (MAS) Promoter}

\author{
Yi $\mathrm{An}^{1 \dagger}$, Ya Geng ${ }^{1 \dagger}$, Junguang Yao ${ }^{1}$, Chun Wang ${ }^{2}$ and Juan $\mathrm{Du}^{3 *}$ \\ ${ }^{1}$ State Key Laboratory of Subtropical Silviculture, School of Forestry and Biotechnology, Zhejiang A\&F University, Hangzhou, \\ China, ${ }^{2}$ State Key Laboratory of Rice Biology, China National Rice Research Institute, Chinese Academy of Agricultural \\ Sciences, Hangzhou, China, ${ }^{3}$ College of Life Sciences, Zhejiang University, Hangzhou, China
}

Gene editing technology in woody plants has great potential for understanding gene function, and altering traits affecting economically and ecologically important traits. Gene editing applications in woody species require a high genome editing efficiency due to the difficulty during transformation and complexities resulting from gene redundancy. In this study, we used poplar 84K (Populus alba $\times$ P. glandulosa), which is a model hybrid for studying wood formation and growth. We developed a new CRISPR/Cas9 system to edit multiple genes simultaneously. Using this system, we successfully knocked out multiple targets of the PHYTOENE DESATURASE 8 in poplar. We found the mutation rate of our CRISPR/Cas9 system is higher (67.5\%) than existing reports in woody trees. We further improved the mutation rate up to $75 \%$ at editing sites through the usage of the mannopine synthase (MAS) promoter to drive Cas9. The MAS-CRISPR/Cas9 is an improved genome-editing tool for woody plants with a higher efficiency and a higher mutation rate than currently available technologies.

Keywords: poplar 84K, CRISPR/Cas9, 35S promoter, MAS promoter, PagPDS gene, high efficiency

\section{INTRODUCTION}

Poplars (Populus) have tremendous economic and ecological value because of their timber, bioenergy applications, rapid pulp production rotation, as well as their key ecological roles in temperate forests across the northern hemisphere (Jansson and Douglas, 2007; Polle et al., 2013). With a modest genome size, high levels of genetic diversity, and a rapid growth rate (Wullschleger et al., 2002), poplars became a model system for woody plants. Hybrid poplars have been genetically modified for studying wood formation, including perennial secondary vascular cambium activity and secondary cell wall deposition (Qiu et al., 2019). Despite existence of some promising approaches for creating knockout mutants in Populus (Fan et al., 2015; Zhou et al., 2015; An et al., 2020a,b; Wang et al., 2020), large-scale gene mutational resources are still lacking for poplars, motivating us to build a robust and high-efficient gene editing system for Populus.

Currently, CRISPR/Cas9 technology is the most effective gene editing technology. In plants, the CRISPR/Cas9 system has not only been used in the study of gene function, but also plays an important role in the improvement of plant traits. Woody plants with long-life spans and outcrossing mating systems are difficult subjects for traditional mutagenesis methods (Bewg et al., 2018). Currently, there are about ten woody plants species that have been successful edited using CRISPR/Cas9 technology: apple (Nishitani et al., 2016), citrus (Jia et al., 2017), grape (Nakajima et al., 2017), cassava (Odipio et al., 2017), cacao (Fister et al., 2018), coffee (Breitler et al., 2018), 
kiwifruit (Wang et al., 2018), parasponia andersonii (Van Zeijl et al., 2018), pomegranate (Chang et al., 2019), and poplar (Fan et al., 2015; Zhou et al., 2015; Wang et al., 2020). Three kinds of Populus have been edited via CRISPR/Cas9, including Populus tomentosa Carr (Fan et al., 2015), 717 (Populus tremula $\times$ P. alba) (Zhou et al., 2015), and Shanxin yang (Populus davidiana $\times$ $P$. bolleana) (Wang et al., 2020). Extensively cultivated in China and Korea, the hybrid clone $84 \mathrm{~K}$ (Populus alba $\times$ P. glandulosa) is a model hybrid for the study wood formation and stress response and also has relatively high rates of transformation compared with other woody plants (Li et al., 2017; Qiu et al., 2019). The recent availability of poplar $84 \mathrm{~K}$ genome sequence facilitates functional genomic studies and the ability to design
CRISPR/CAS9 approaches to target specific genes or even multiple genes (Huang et al., 2020). Therefore, it is of high priority to develop a high-efficiency gene editing system in the $84 \mathrm{~K}$ poplar genotype.

To develop a simple and efficient gene-editing system for poplar $84 \mathrm{~K}$, and for editing multiple genes, we used the existing conventional isocaudomer technique using constructs based on pC1300-Cas9 (binary vector) and SK-gRNA (intermediate vector driven by AtU6-26). The system has the potential to edit an unlimited number of target genes simultaneously (Wang et al., 2015). The promoter is the key to driving the expression of transformed genes. Previous studies conducted on tobacco and maize indicated that the mannopine synthase
A

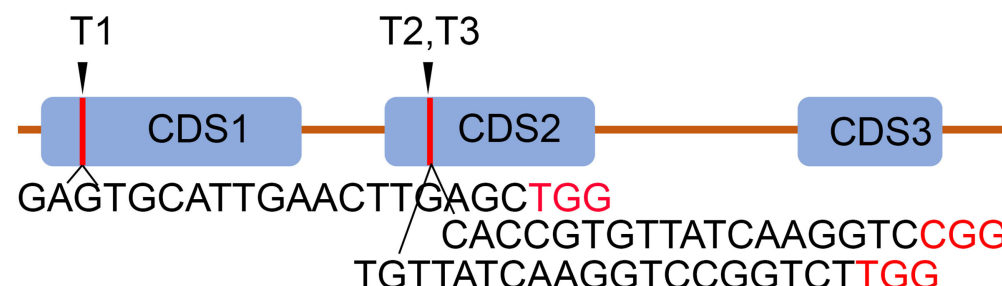

B
GTTTGCAGGGCTGTTGTTACAGTTGTTATAGCTGTTGAATTTGGTTTTGGAGAAATGAGT 60 GTTTGCAGGGCTGTTGTTACAGTTGTTATAGCTGTTGAATTTGGTTTTGGAGAAATGAGT 60

PAM

PagPDS form P. glandulosa

PagPDS form P. alba

PagPDS form P. glandulosa

PagPDS form P. alba

PagPDS form P. glandulosa

PagPDS form $P$. alba

PagPDS form P. glandulosa

PagPDS form $P$. alba

PagPDS form P. glandulosa

PagPDS form $P$. alba

PagPDS form P. glandulosa

PagPDS form $P$. alba

PagPDS form P. glandulosa PagPDS form P. alb

PagPDS form $P$. glandulosa PagPDS form $P$. alba

GCATTGAACTTGAGCTGGCATAGTAAATCATTAGACTCTCAAGTTGCCTTGAGATGTGGC 120 GCATTGAACTTGAGCTGGCATAGTAAATCATTAGACTCTCAAGTTGCCTTGAGATGTGGC 120

GCTTATCCTACTTGTTCTCACCAAACGAATGCACTAGCTTTTAGAGGCAGTGAATCAATG 180 GCTTATCCTACTTGTTCTCACCAAACGAATGCACTAGCTTTTAGAGGCAGTGAATCAATG 180

GGCCATTCTTTGAAATTCCCATTTGGAAATTCTTCTGCTAAAACAAGACTAAGGAATCAT 240 GGCCATTCTTTGAAATTCCCATTTGGAAATTCTTCTGCTAAAACAAGACTAAGGAATCAT 240

ATCCGCCCTCCTTTGCGGGTGCGTAGTTCTCTACACTACAGGGTAATTATTAGTTGCCAA 300 ATCCGCCCTCCTTTGCGGGTGCGTAGTTCTCTACACTACAGGGTAATTATTAGTTGCCAA 300

TCAATACGTGAAAATTTGGGGTGATCTTTTGTCTACGCTGTAGGTTGTCTGTATGGACTA 360 TCAATACGTGAAAATTTGGGGTGATCTTTTGTCTACGCTGTAGGTTGTCTGTATGGACTA 360 PAM PAM TCCAAGACCGGACCTTGATAACACGGTGAATTTCTTAGAGGCTGCCTTGTTATCTTCATC 420 TCCAAGACCGGACCTTGATAACACGGTGAATTTCTTAGAGGCTGCCTTGTTATCTTCATC 420

CTTTCGTTCTTCTCCGCGTCCAGCTAAACCATTAAATGTTGTCATTGCTGGTGCAGGTGA 480 CTTTCGTTCTTCTCCGCGTCCAGCTAAACCATTAAATGTTGTCATTGCTGGTGCAGGTGA 480

TGAAATCTTATCCTTTTTTTGTATGGGAAAAAACTGTGTTGATTATTTAGATTGATTTCT 540 TGAAATTTATCCTTTTTTTGTATGGAAAAACTGTGTTGATTATTTAGATTGATTTCT 540

FIGURE 1 | Schematic diagrams illustrating the PagPDS target sites and PagPDS gene sequence alignment. (A) Schematic diagrams illustrating the PagPDS target sites (T1-T3) and their sequences. The PAM (5'-NGG-3') are denoted in red. Blue boxes indicate exons (exon1-exon4). Orange lines indicate introns. (B) PagPDS genomic sequences (exon1-exon2) from $84 \mathrm{~K}$ (Populus alba $\times$ P. glandulosa). Blue lines indicate exons, letters in red indicate PAM sequence, highlighted indicate the 3 target sites. 
(MAS) promoter could increase the direct expression of $\beta$ glucuronidase activity to 2-20 fold compared with the commonly used enhanced CaMV 35S promoter (Ni et al., 1995; Lee et al., 2007). We hypothesized that MAS promoter could similarly drive Cas9 to enhance editing events in $84 \mathrm{~K}$. Therefore, we constructed a new gene editing system which used 35S/MAS promoter to drive Cas9 and studied the editing efficiency in poplar $84 \mathrm{~K}$. The new system successfully editing multiple targets of phytoene desaturase gene 8 (PDS) with high mutation rates.

A

\section{SK-gRNA}

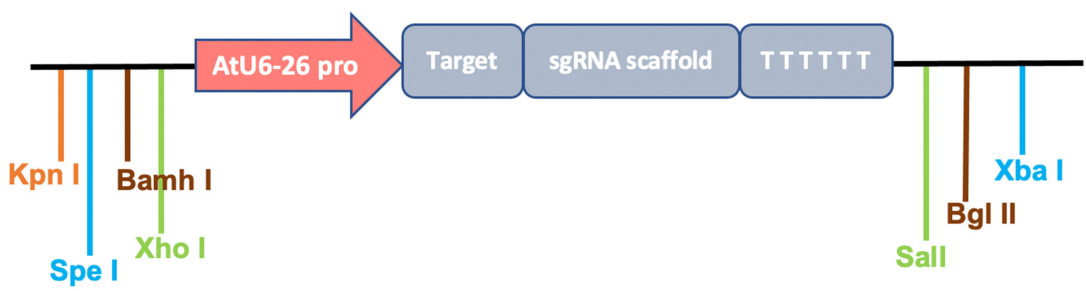

\section{pC1300-Cas9}

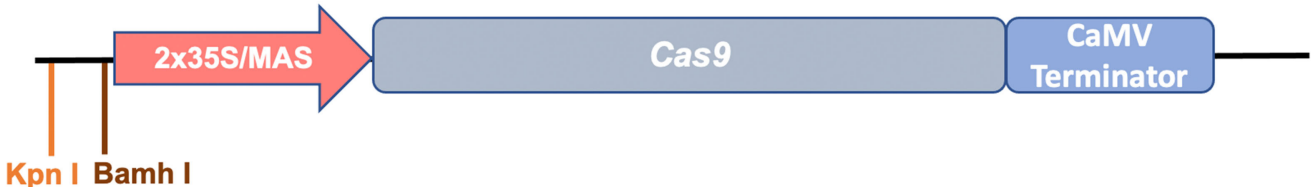

B
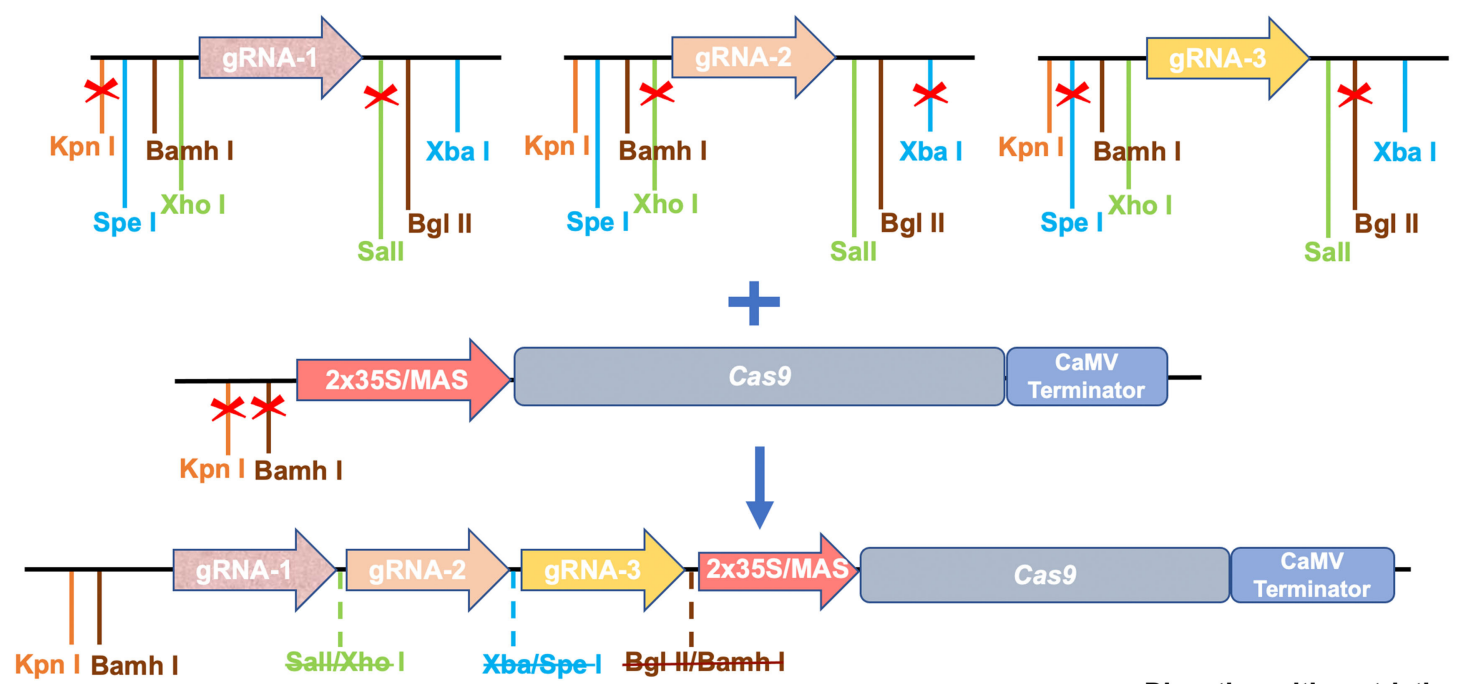

Bamh-1

C

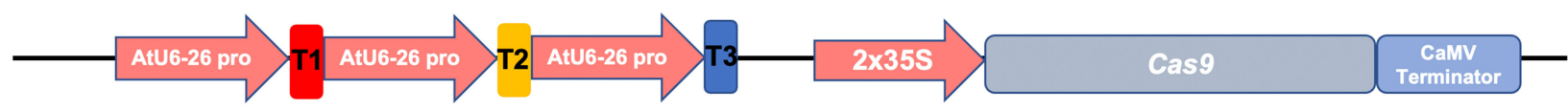

FIGURE 2 | Schematic diagram illustrating the structure of vectors and construction strategy. (A) The intermediate vector SK-gRNA contains the U6 promotor, an sgRNA scaffold, and the terminator. The restriction sites used for cloning are indicated. Binary vector pC1300-Cas 9 contains the $2 \times 35 \mathrm{~S}$ or MAS promotor, a codon-optimized Cas9 coding sequence, and the CaMV terminator. (B) Vector construction strategy. Kpn I and BamH I sites are indicated in front of the $2 \times 35 \mathrm{~S}$ or MAS promotor. Three gRNA scaffolds with PagPDS targets are, respectively, digested (contains Kpn I, BamH I, Xho I, Sal I, Xba I, and Spel I) and cloned into pC1300-Cas9 between the Kpn I and BamH I sites in a one-step ligation. (C) Structure of destination vectors used in the CRISPR/Cas9 system. 


\section{MATERIALS AND METHODS \\ Cloning of PagPDS Fragments and Selection of Target Site}

Wide-type (WT) poplar $84 \mathrm{~K}$ genomic DNA was extracted by the cetyltrimethylammonium bromide (CTAB) method (Fan et al., 2015). The phytoene desaturase gene (PDS) was cloned from extracted genomic DNA. Briefly, primers were designed against PagPDS from genome sequence of poplar $84 \mathrm{~K}$. The primers are PagPDS_F: GTTTGCAGGGCTGTTGTTACAGTT and PagPDS_R: CATTTAATGGTGCAGGGAGAACTTCAG. The amplification reaction was conducted at $95^{\circ} \mathrm{C}$ for $5 \mathrm{~min}, 35$ cycles at $95^{\circ} \mathrm{C}$ for $30 \mathrm{~s}, 58^{\circ} \mathrm{C}$ for $30 \mathrm{~s}, 72^{\circ} \mathrm{C}$ for $35 \mathrm{~s}$, and $72^{\circ} \mathrm{C}$ for $10 \mathrm{~min}$. The amplicon product was electrophoresed on an ethidium bromide-stained agarose gel (1\%). DNA was extracted from gel using the TIANgel Midi Purification Kit (Tiangen, Beijing, China) and cloned into the pMD18-T Simple vector and then confirmed by Sanger sequencing. The single-guide RNA (sgRNA) sequence for PDS was designed (Figure 1A) based on the allelic variation of PagPDS in Figure 1B. Three sgRNAs were designed to target three conserved sites optimizing GC content and PAM sequence.

\section{Vector Construction}

The system is comprised of two vectors, the first of which is pC1300-Cas9, which was based on the pCAMBIA1300 backbone, which has been frequently used for Agrobacteriummediated transformation in Populus. The vector contains the codon-optimized Streptococcus pyogenes Cas9 gene driven by the 2xCaMV35S promoter. pC1300-Cas9 contains Kpn I and $\mathrm{BamH}$ I restriction enzyme sites, which are designed for allowing integration of gRNA cassettes. Then, we developed novel vectors based on pC1300-Cas9 backbone, introducing the Superpromoter to drive Cas9 expression, with the aim to increase the expression level of Cas9 in the Populus cells. The Superpromoter is a synthetic promoter consists of a trimer of the octopine synthase transcriptional activating element affixed to the mannopine synthase2\# transcriptional activating element plus minimal promoter (Lee et al., 2007). The MAS promoter sequence is shown in Supplementary Sequence 1.

Three sgRNAs guiding sequences were inserted into the SKgRNA vector (Wang et al., 2015) between the two Aar I sites using annealed oligonucleotides (we named the resulting three vectors SK-gRNA1, SK-gRNA2, and SK-gRNA3). The pC1300Cas9 vector contains the codon-optimized Streptococcus pyogenes Cas9 gene and the Kpn I and BamH I isocaudomer enzyme sites, which are designed to allow integration of gRNA cassettes. The SK-gRNA vector also contains isocaudomer restriction enzyme sites, namely Spel/ Xba I, Xho I/Sal I, and BamH I/BgI II sites (Figure 2A). The steps of vector construction are shown in Figure 2B. The three vectors (SK-gRNA1, SKgRNA2, and SK-gRNA3) were digested separately with restriction enzymes, and then inserted into the pC1300-2 $\times 35$ S-Cas 9
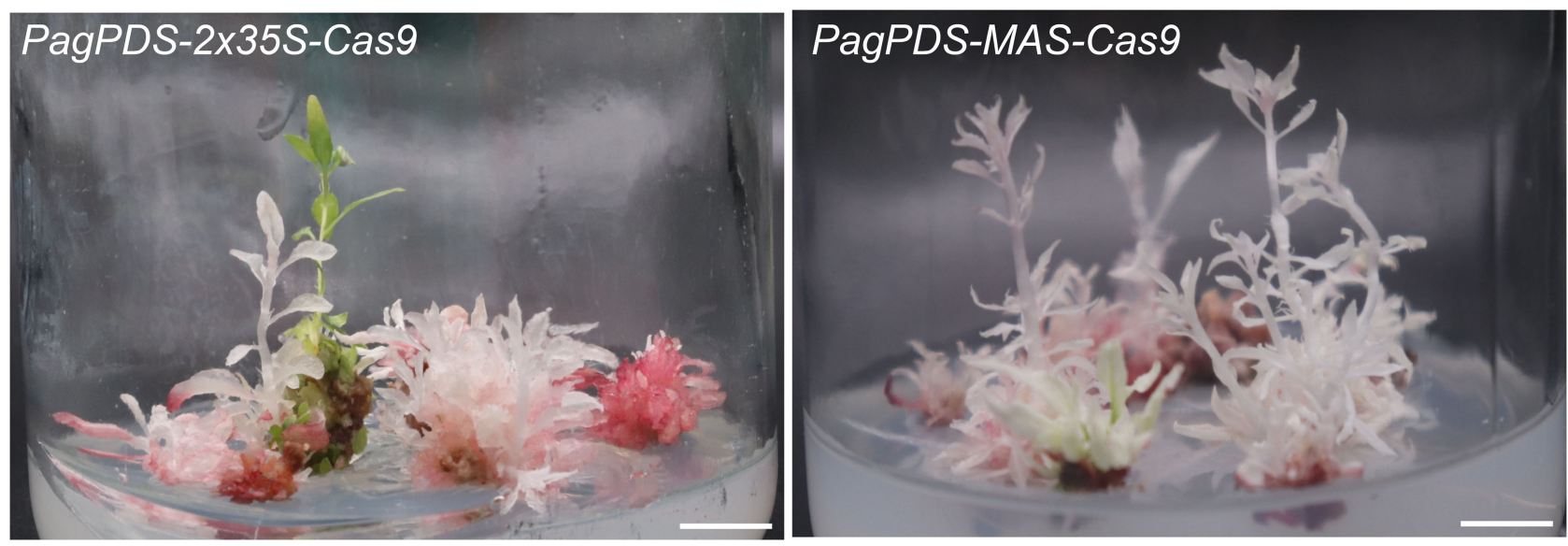

FIGURE 3 | Phenotypes of the PagPDS-2 × 35S/MAS-Cas9-mediated transgenic plants. Scale bar $=1 \mathrm{~cm}$.

TABLE 1 | Determination of mutation rate in transgenic To poplar plants generated with the PagPDS-2 × 35S/MAS-Cas9 system.

\begin{tabular}{|c|c|c|c|c|c|c|c|}
\hline \multirow[t]{2}{*}{ Target gene } & \multirow[t]{2}{*}{ Number of plants examined } & \multirow[t]{2}{*}{ Number of plants with mutations } & \multirow[t]{2}{*}{ Mutation rate $(\%)$} & \multicolumn{2}{|c|}{ Albinism phenotype } & \multicolumn{2}{|c|}{ Pale green phenotype } \\
\hline & & & & Number & $\%$ & Number & $\%$ \\
\hline PagPDS-2 × 35S-Cas9 & 40 & 27 & 67.5 & 21 & 77.8 & 6 & 22.2 \\
\hline PagPDS-MAS-Cas9 & 40 & 30 & 75 & 23 & 76.7 & 7 & 23.3 \\
\hline CK & 40 & 0 & 0 & ND & ND & ND & ND \\
\hline
\end{tabular}

CK, Empty vector; ND, not determined. 
and pC1300-MAS-Cas9 binary vector between the Kpn I and $B a m H$ I sites by one-step ligation (Wang et al., 2015). The constructed vectors were named PagPDS-2 × 35S-Cas9, and PagPDS-MAS-Cas9, respectively. The final vector diagram is shown in Supplementary Figure 1 and the Cas9 sequence in
Supplementary Sequence 2. The construct was sequenced and the expected sequences confirmed.

\section{Transformation and Regeneration}

The vector containing CRISPR/Cas9 with gRNA expression

A

\begin{tabular}{|c|c|c|c|}
\hline \multicolumn{4}{|c|}{ PagPDS-2x35S-Cas9 } \\
\hline \multirow[t]{3}{*}{ phenotype } & target 1 & target 2 & \\
\hline & GGAGAAATGAGTGCATTGAACTTG-AGCTGGCATAG & $\begin{array}{c}\text { АСTATCCAAGACCGGACCTTGATAACACGGTGAATTTC } \\
\text { target } 3\end{array}$ & \\
\hline & & АСТАTCCAAGACCGGACCTTGATAACACGGTGAATTTC & \\
\hline \multirow[t]{2}{*}{ albinism } & GGAGAAATGAGTGCATTGAACTTGTAGCTGGCATAG & АСТАTCCA-----GGACCTTGATAACACGGTGAATTTC & $+1 /-5 \times 1$ \\
\hline & GGAGAAATGAGTGCATTGAACTTGTAGCTGGCATAG & АCTATCCA-----GGACCTTGATAACACGGTGAATTTC & $+1 /-5$ \\
\hline \multirow[t]{2}{*}{ albinism } & GGAGAAATGAGTGCATTGAACTTGAAGCTGGCATAG & АСТАTCCAA-----GACCTTGATAACACGGTGAATTTC & $+1 /-5 \times 1$ \\
\hline & GGAGAAATGAGTGCATTGAACTTGAAGCTGGCATAG & АСТАTCCAA-----GACCTTGATAACACGGTGAATTTC & $+1 /-5$ \\
\hline \multirow[t]{2}{*}{ albinism } & GGAGAAATGAGTGCATTGAACTTGTAGCTGGCATAG & АСTATCCAAGA--GGACCTTGATAAAGACGGTGAATTTC & $+3 /-4 \times 1$ \\
\hline & GGAGAAATGAGTGCATTGAACTTGTAGCTGGCATAG & АСТАTCCA-----GGACCTTGATAACACGGTGAATTTC & $+1 /-5$ \\
\hline \multirow[t]{2}{*}{ albinism } & GGAGAAATGAGTGCATTGAACTTGTAGCTGGCATAG & АСТАTCCA------GGACCTTGATAACACGGTGAATTTC & $+1 /-5 \times 2$ \\
\hline & GGAGAAATGAGTGCATTGAACTTGTAGCTGAC & СТАTCCAAGA--GGACCTTGATAACACGGTGAATTTC & $+3 /-4$ \\
\hline \multirow[t]{2}{*}{ albinism } & GGAGAAATGAGTGCATTGAACTTGTAGCTGGCATAG & АСТАTCCA-----GGACCTTGATAACACGGTGAATTTC & $+1 /-5 \times 15$ \\
\hline & GGAGAAATGAGTGCATTGAACTTGTAGCTGGCATAG & АСTATCCAAGA--GGACCTTGATAACACGGTGAATTTC & $+1 /-2$ \\
\hline \multirow[t]{2}{*}{ albinism } & GGAGAAATGAGTGCATTGAA------------------ & ----------------CTTGATAACACGGTGAATTTC & $-305 \times 1$ \\
\hline & GGAGAAATGAGTGCATTGAA------------------ & --------------CTTGATAACACGGTGAATTTC & -305 \\
\hline \multirow[t]{2}{*}{ pale } & GGAGAAATGAGTGCATTGAACTTGTAGCTGGCATAG & АСТАTCCA-----GGACCTTGATAACACGGTGAATTTC & $+1 /-5 \times 3$ \\
\hline & GGAGAAATGAGTGCATTGAACTTG-AGCTGGCATAG & АСТАTCCAAGA--GGACCTTGATAACACGGTGAATTTC & -2 \\
\hline \multirow[t]{2}{*}{ pale } & GGAGAAATGAGTGCATTGAACTTG-AGCTGGCATAG & АСTATCCAAGA--GGACCTTGATAACACGGTGAATTTC & -2 \\
\hline & GGAGAAATGAGTGCATTGAACTTG-AGCTGGCATAG & АСТАTCCAAGA--GGACCTTGATAACACGGTGAATTTC & -2 \\
\hline \multirow[t]{2}{*}{ pale } & GGAGAAATGAGTGCATTGAACTTGTAGCTGGCATAG & АСTATCCAAGA--GGACCTTGATAACACGGTGAATTTC & $+1 /-2 \times 2$ \\
\hline & GGAGAAATGAGTGCATTGAACTTG-AGCTGGCATAG & АСТАTCCAAGACCGGACCTTGATAACACGGTGAATTTC & - \\
\hline \multirow[t]{2}{*}{ green } & GGAGAAATGAGTGCATTGAACTTG-AGCTGGCATAG & АСTATCCAAGACCGGACCTTGATAACACGGTGAATTTC & - \\
\hline & GGAGAAATGAGTGCATTGAACTTG-AGCTGGCATAG & АСTATCCAAGACCGGACCTTGATAACACGGTGAATTTC & - \\
\hline
\end{tabular}

B

PagPDS-MAS-Cas9

phenotype

target1

GGAGAAATGAGTGCATTGAACTTGAGCTGGCATAG

albinism GGAGAAATGAGTGCATTGAA-

GGAGAAATGAGTGCATTGAA---------------

albinism GGAGAAATGAGTGCATTGAACTTG----------GGAGAAATGAGTGCATTGAACTTG------------

albinism GGAGAAATGAGTGCATTGAACTTG---------GGAGAAATGAGTGCATTGAACTTG-----------

albinism GGAGAAATGAGTGCATTGAACTTGTAGCTGGCATAG GGAGAAATGAGTGCATTGAACTTGTAGCTGGCATAG

albinism GGAGAAATGAGTGCATTGAACTTGTAGCTTGG-ATAG GGAGAAATGAGTGCATTGAACTTGT-CTTGGATAG

albinism GGAGAAATGAGTGCATCGAACTT-AGCTGGCATAG GGAGAAATGAGTGCATTGAACTTGGGTCCGGTCTTG

albinism GGAGAAATGAGTGCATTGAACTT-AGCTGGCATAG GGAGAAATGAGTGCATTGAACTT-AGCTGGCATAG

albinism GGAGAAATGAGTGCATTGAACTTG GGAGAAATGAGTGCATTGAACTTGTAGCTGGCATAG

pale GGAGAAATGAGTGCATTGAACTTGTAGCTGGCATAG GGAGAAATGAGTGCATTGAACTTGAGCTGGCATAG

pale GGAGAAATGAGTGCATTGAACTTGGGAGAAATGAGTGCATTGAACTTGAGCTGGCATAG green GGAGAAATGAGTGCATTGAACTTGAGCTGGCATAG GGAGAAATGAGTGCATTGAACTTGAGCTGGCATAG target2

ACTATCCAAGACCGGACCTTGATAACACGGTGAATTTC target 3

ACTATCCAAGACCGGACCTTGATAACACGGTGAATTTC

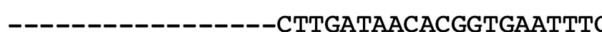
----------------CTTGATAACACGGTGAATTTC ----------------CCGGATAACACGGTGAATTTC ----------------CCGGATAACACGGTGAATTTC ----------------CTTGATAACACGGTGAATTTC ----------------CTTGATAACACGGTGAATTTC ACTATCCAAGACCG-GGATTTC ACTATCCAAGACCG -GGATTTC

ACTATGCCAGCTCGGACCTTGATAACACGGTGAATTTC ACTATGCCAGCTCGGACCTTGATAACACGGTGAATTTC ACTATCCAAGA-CGGACCTTGATAACACGGTGAATTTC $-3+1 \quad x 1$ ACTATGC-----CAGCTCTTGATAACACGGTGAATTTC -18+13 ACTATCCAAGA-CGGACCTTGATAACACGGTGAATTTC $-2 \quad x 1$ ACTATCCAAGA-CGGACCTTGATAACACGGTGAATTTC -2 --------------CTTGATAACACGGTGAATTTC -301 x1 ACTATCCA-ACCGGACTCTTGATAACACGGTGAATTTC $-2 /+1$ АСTATCCA-----GGACCTTGATAACACGGTGAATTTC -6/+1 x4 ACTATCCAAGACCGGACCTTGATAACACGGTGAATTTC -------------CTTGATAACACGGTGAATTTC ACTATCCAAGACCGGACCTTGATAACACGGTGAATTTC ACTATCCAAGACCGGACCTTGATAACACGGTGAATTTC ACTATCCAAGACCGGACCTTGATAACACGGTGAATTTC

\begin{tabular}{ll}
-305 & $x 15$ \\
-305 & \\
$303 /+$ & $x 2$ \\
$303 /+2$ & \\
-301 & $x 1$ \\
-301 & \\
$-16+2$ & $x 1$ \\
$-16+2$ & \\
$-5+4$ & $x 1$ \\
$-2+1$ & \\
$-3+1$ & $x 1$ \\
$-18+13$ & \\
-2 & $x 1$ \\
-2 & \\
-301 & $x 1$ \\
$-2 /+1$ & \\
$-6 /+1$ & $x 4$ \\
- & \\
-301 & $\times 3$ \\
- & \\
- & $x 10$ \\
- & \\
\hline
\end{tabular}
$\mathrm{x}$ $01 \times 1$ $\mathrm{x} 1$ $\mathbf{x} 1$ 
TABLE 2 | Summary of the mutation types at each target site.

\begin{tabular}{|c|c|c|c|c|}
\hline & \multicolumn{2}{|c|}{ PagPDS-2 × 35S-Cas9 } & \multicolumn{2}{|c|}{ PagPDS-MAS-Cas9 } \\
\hline & T1 & T2/T3 & T1 & T2/T3 \\
\hline Biallelic mutation & 1 & 21 & 3 & 2 \\
\hline Homozygous & 20 & 4 & 19 & 20 \\
\hline Heterozygous & 5 & 2 & 7 & 7 \\
\hline Unmodified & 14 & 13 & 11 & 11 \\
\hline Mutation rate & $65 \%$ & $67.5 \%$ & $72.5 \%$ & $72.5 \%$ \\
\hline
\end{tabular}

cassettes was then transformed into the Agrobacterium tumefaciens strain GV3101 (Han et al., 2000; Li et al., 2017; An et al., 2020b). Briefly, Agrobacterium cells harboring the vectors were harvested by centrifugation and then resuspended to $\mathrm{OD}_{600}$ $=0.3-0.4$. Poplar $84 \mathrm{~K}$ leaf disks were soaked for $20 \mathrm{~min}$ on a shaker with the resuspended cells at room temperature. The inoculated leaf disks were co-cultivated at $22^{\circ} \mathrm{C}$ in the dark for 2 to 3 days. The leaf discs were then washed with sterile doubledistilled water and cultured on a callus-induction medium for 10 to 30 days in the dark. The transgenic lines were selected by culturing on medium supplemented with hygromycin $(2.0$ $\mathrm{mg} / \mathrm{L})$. The gene-edited plants were vegetatively propagated in half-strength Murashige and Skoog medium ( $\mathrm{pH}$ 5.7) containing $0.8 \%(\mathrm{w} / \mathrm{v})$ agar at room temperature under the light intensity of $50 \mu \mathrm{mol} \mathrm{m}^{-2} \mathrm{~s}^{-1}$ and a 16/8 h (light/dark) photoperiod.

\section{Detection of Mutations}

For analysis of the mutations of edited PagPDS in transgenic $\mathrm{T}_{0}$ plants, genomic DNA was extracted from stable transgenic or wild-type plants using CTAB as described above. The genomic DNAs were used as templates to amplify the endogenous PagPDS fragment by Polymerase Chain Reaction (PCR). The primers used for PCR were PagPDS_Mut_F: AACTGGGTATGCGAAGACTTCC and PagPDS_Mut_R: GATTTCATCACCTGCACCAGCAAT. The primers amplified the region spanning the three target sites of the Cas9 system (target 1: 5'-GAGTGCATTGAACTTGAGCTGG-3'; target 2: $5^{\prime}$-CCAAGACCGGACCTTGATAACA-3'; target $3: 5^{\prime}$ CCGGACCTTGATAACACGGTGA-3'). The PCR products were amplified and cloned into the pMD18-T Simple vector as mentioned above for Sanger sequencing to evaluate the editing outcomes of CRISPR/Cas9 transfection. Finally, individual sequences were aligned to the wild-type sequence using SnapGene to determine the mutations effects on predicted modified peptide sequences.

\section{RESULTS}

\section{CRISPR/Cas9 Targeted Mutagenesis in PagPDS}

To establish a new CRISPR/Cas9 genome editing system in poplars, we cloned the phytoene desaturase gene 8 from the hybrid poplar $84 \mathrm{~K}$ and selected three target sites with $5^{\prime}$-NGG$3^{\prime}$ PAMs of two exons. We named the three target sites T1, T2, and T3 (Figure 1A). The multiple CRISPR RNA (crRNA) cassettes were driven by the Arabidopsis RNA polymerase III promoter AtU6-26 while Cas9 was driven by the $2 \times 35$ S promoter or MAS promoter in pCambial300 binary vectors (Figure 2A). The target sequence was confirmed by amplification and sequencing. The three gRNAs were inserted into the pC1300-2 × 35S-Cas9 and pC1300-MAS-Cas9 binary vectors between the Kpn I and BamH I sites by a one-step ligation (Figure 2B). Two constructs (Figure 2C) were introduced into the poplar $84 \mathrm{~K}$ via Agrobacterium-mediated transformation. The transgenic plants were selected by culture on $1 / 2$ MS medium supplemented with hygromycin and grown with a light and dark cycles of $16 / 8 \mathrm{~h}$.

\section{Phenotypes of PagPDS-2 x 35S-Cas9 Transgenic Plants}

Forty positive candidate transgenic lines were obtained for PagPDS-2 $\times$ 35S-Cas9 and confirmed by DNA PCR amplification. Both biallelic homozygous and heterozygous mutants of PDS are known to exhibit albino phenotypes (Fan et al., 2015). Here, albino seedlings were observed, confirming that mutations were generated at the PDS gene (Figure 3). To evaluate the simultaneous editing of multiple target sites, the genomic DNA of 40 transgenic plants was extracted independently, and the targeted sequences of $P D S$ were amplified by PCR. The sequencing results showed 67.5\% (27 out of 40) mutation rate. Out of 40 albino seedlings, $77.8 \%$ (21 out of 27) were albinos and $22.2 \%$ (6 out of 27) showed pale green phenotype (Table 1). At the T1 site, all seedlings carried mutations, and $53.75 \%$ of the mutations were a mononucleotide insertion. At T2/T3 loci, deletions were noted ranging from 2 to 5 nt near the PAM. Gene editing data are presented in Figure 4A. At the T1 locus, five plants had a mutation in one allele while 21 plants had mutations in both alleles, including 20 homozygous mutations and 1 biallelic mutations. At T2/T3 loci, the mutation rate was $67.5 \%$ (27 out of 40 ), including 21 biallelic mutations and four homozygous mutations (Table 2).

\section{Phenotypes of PagPDS-MAS-Cas9 Transgenic Plants}

To explore the activity of the MAS promoter in poplars, we used the same method to construct a vector with MAS driving expression of Cas9. This construct was introduced into poplar $84 \mathrm{~K}$ resulting in 40 transgenic lines, of which30 were albino. In the MAS-Cas9 system, the mutation rate was 75\% (30 out of 
40 ), which was higher than that of the $2 \times 35$ S-Cas9 system. $76.7 \%$ (23 of 30 ) of mutant plants were albinos and $23.3 \%$ (7 out of 30) showed a pale green phenotype (Table 1). Sequencing of the albino plants was used to define the resulting mutations. Large deletions were found between T1 and T2/T3 (Figure 4B). At mutation site T1, 29 plants $(72.5 \%)$ had at least one allele edited while 22 plants had the edited mutations in both alleles, including 19 homozygous mutations and 3 biallelic mutations. At the T2/T3 loci, the mutation rate was $72.5 \%$, including 20 homozygous mutations and 2 biallelic mutations (Table 2 ).

\section{DISCUSSION}

In this study, we established a simplified method for creating mutations with high frequency directly in the model poplar $84 \mathrm{~K}$ genotype. We used two vectors to construct an engineered binary vector based on the classical isocaudomer technique to simplify the procedures. With appropriate restriction enzyme sites, as many as three gRNAs or more can be assembled together in one step in a single ligation reaction, which enables creating mutations in multiple target sites within a single plant. This system both simplifies the procedures for gene editing, and also increases the frequency of mutations.

Our ability to successfully target multiple sites of the PagPDS genes in Populus make demonstrates the ability to edit multiple genes at the same time, including duplicated homologous genes. Gene duplication is common in poplar and other trees, so the simple system could be applied to edit all homologs in trees. In order to better compare the editing efficiency with the binary pYLCRIPSR/Cas9, three target sites were selected using the criteria of Fan et al. (2015). The mutation rate in our system is improved to higher rate, $67.5 \%$ in CaMV $35 \mathrm{~S}$ promoterbased system and 75\% in pC1300-MAS-Cas9 system than the reported mutation rate (51.7\%) in Populus tomentosa Carr (Fan et al., 2015). Indeed in our pC1300-MAS-Cas9 system, there is high editing efficiency, which is of practical importance as it reduces the work and effort of producing and evaluating enough transgenic lines to recover desired mutations. Based on previous studies, the MAS promoter increases the expression of gene fusions in tobacco and maize (Ni et al., 1995; Lee et al., 2007).

\section{REFERENCES}

An, Y., Geng, Y., Yao, J., Fu, C., Lu, M., Wang, C., et al. (2020a). Efficient genome editing in populus using CRISPR/Cas12a. Front. Plant Sci. 11:593938. doi: 10.3389/fpls.2020.593938

An, Y., Zhou, Y., Han, X., Shen, C., Wang, S., Liu, C., et al. (2020b). The GATA transcription factor GNC plays an important role in photosynthesis and growth in poplar. J. Exp. Bot. 71, 1969-1984. doi: 10.1093/jxb/erz564

Bewg, W. P., Ci, D., and Tsai, C. J. (2018). Genome editing in trees: from multiple repair pathways to long-term stability. Front. Plant Sci. 9:1732. doi: 10.3389/fpls.2018.01732

Breitler, J.-C., Dechamp, E., Campa, C., Zebral Rodrigues, L. A., Guyot, R., Marraccini, P., et al. (2018). CRISPR/Cas9-mediated efficient targeted mutagenesis has the potential to accelerate the domestication of Coffea canephora. Plant Cell Tissue Organ. Cult. 134, 383-394. doi: $10.1007 /$ s11240-018-1429-2
It is thus likely that the MAS promoter promoted the expression of Cas9 in our experiments in poplar, enriching the variety and efficiency of edits.

Our a high-efficiency genome-editing tool of pC1300-MASCas9 will facilitate gene functional research in woody tree species. In future studies we will directly test whether the PC1300-MASCas9 has high editing efficiency in other woody plants and trees.

\section{DATA AVAILABILITY STATEMENT}

The original contributions presented in the study are included in the article/Supplementary Material, further inquiries can be directed to the corresponding author/s.

\section{AUTHOR CONTRIBUTIONS}

YA, JD, and CW designed the research and wrote the paper. YA, CW, JY, and YG performed the research. YA and JY analyzed data. All authors reviewed the manuscript.

\section{FUNDING}

This research was supported by National Key Program on Transgenic Research (2018ZX08020002) to Zhejiang University (JD). It was supported by the by the National Natural Science Foundation of China (31901327) to YA.

\section{ACKNOWLEDGMENTS}

We thank Dr. Andrew Groover for help with editing the manuscripts.

\section{SUPPLEMENTARY MATERIAL}

The Supplementary Material for this article can be found online at: https://www.frontiersin.org/articles/10.3389/fpls.2021. 703546/full\#supplementary-material 
Huang, X., Chen, S., Peng, X., Bae, E.-K., Dai, X., Liu, G., et al. (2020). An improved draft genome sequence of hybrid Populus alba $\times$ Populus glandulosa. J. For. Res 32, 1663-1672. doi: 10.1007/s11676-020-01235-2

Jansson, S., and Douglas, C. J. (2007). Populus: a model system for plant biology. Annu. Rev. Plant Biol. 58, 435-458. doi: 10.1146/annurev.arplant.58.032806.103956

Jia, H., Xu, J., Orbovic, V., Zhang, Y., and Wang, N. (2017). Editing citrus genome via SaCas9/sgRNA system. Front. Plant Sci. 8:2135. doi: $10.3389 /$ fpls.2017.02135

Lee, L. Y., Kononov, M. E., Bassuner, B., Frame, B. R., Wang, K., and Gelvin, S. B. (2007). Novel plant transformation vectors containing the superpromoter. Plant Physiol. 145, 1294-1300. doi: 10.1104/pp.107.106633

Li, S., Zhen, C., Xu, W., Wang, C., and Cheng, Y. (2017). Simple, rapid and efficient transformation of genotype Nisqually-1: a basic tool for the first sequenced model tree. Sci. Rep. 7:2638. doi: 10.1038/s41598-017-02651-x

Nakajima, I., Ban, Y., Azuma, A., Onoue, N., Moriguchi, T., Yamamoto, T., et al. (2017). CRISPR/Cas9-mediated targeted mutagenesis in grape. PLoS ONE 12:e0177966. doi: 10.1371/journal.pone.0177966

Ni, M., Cui, D., Einstein, J., Narasimhulu, S., Vergara, C. E., and Gelvin, S. B. (1995). Strength and tissue specificity of chimeric promoters derived from the octopine and mannopine synthase genes. Plant J. 7, 661-676. doi: 10.1046/j.1365-313X.1995.7040661.x

Nishitani, C., Hirai, N., Komori, S., Wada, M., Okada, K., Osakabe, K., et al. (2016). Efficient genome editing in apple using a CRISPR/Cas9 system. Sci. Rep. 6:31481. doi: 10.1038/srep31481

Odipio, J., Alicai, T., Ingelbrecht, I., Nusinow, D. A., Bart, R., and Taylor, N. J. (2017). Efficient CRISPR/Cas9 genome editing of phytoene desaturase in cassava. Front. Plant Sci. 8:1780. doi: 10.3389/fpls.2017.01780

Polle, A., Janz, D., Teichmann, T., and Lipka, V. (2013). Poplar genetic engineering: promoting desirable wood characteristics and pest resistance. Appl. Microbiol. Biotechnol. 97, 5669-5679. doi: 10.1007/s00253-013-4940-8

Qiu, D., Bai, S., Ma, J., Zhang, L., Shao, F., Zhang, K., et al. (2019). The genome of Populus alba $\times$ Populus tremula var. glandulosa clone 84K. DNA Res. 26, 423-431. doi: 10.1093/dnares/dsz020
Van Zeijl, A., Wardhani, T. A. K., Seifi Kalhor, M., Rutten, L., Bu, F., Hartog, M., et al. (2018). CRISPR/Cas9-Mediated mutagenesis of four putative symbiosis genes of the tropical tree parasponia andersonii reveals novel phenotypes. Front. Plant Sci. 9:284. doi: 10.3389/fpls.2018.0 0284

Wang, C., Shen, L., Fu, Y., Yan, C., and Wang, K. (2015). A simple CRISPR/Cas9 system for multiplex genome editing in rice. J. Genet. Genomics 42, 703-706. doi: 10.1016/j.jgg.2015.0 9.011

Wang, J., Wu, H., Chen, Y., and Yin, T. (2020). Efficient CRISPR/Cas9-mediated gene editing in an interspecific hybrid poplar with a highly heterozygous genome. Front. Plant Sci. 11:996. doi: 10.3389/fpls.2020.00996

Wang, X., Tu, M., Wang, D., Liu, J., Li, Y., Li, Z., et al. (2018). CRISPR/Cas9mediated efficient targeted mutagenesis in grape in the first generation. Plant Biotechnol. J. 16, 844-855. doi: 10.1111/pbi.12832

Wullschleger, S. D., Jansson, S., and Taylor, G. (2002). Genomics and forest biology: Populus emerges as the perennial favorite. Plant Cell 14, 2651-2655. doi: $10.1105 /$ tpc. 141120

Zhou, X., Jacobs, T. B., Xue, L. J., Harding, S. A., and Tsai, C. J. (2015). Exploiting SNPs for biallelic CRISPR mutations in the outcrossing woody perennial Populus reveals 4-coumarate:CoA ligase specificity and redundancy. New Phytol. 208, 298-301. doi: 10.1111/nph.13470

Conflict of Interest: The authors declare that the research was conducted in the absence of any commercial or financial relationships that could be construed as a potential conflict of interest.

Copyright (C) 2021 An, Geng, Yao, Wang and Du. This is an open-access article distributed under the terms of the Creative Commons Attribution License (CC BY).

The use, distribution or reproduction in other forums is permitted, provided the original author(s) and the copyright owner(s) are credited and that the original publication in this journal is cited, in accordance with accepted academic practice. No use, distribution or reproduction is permitted which does not comply with these terms. 